\title{
Antioxidant activity and phenolic content of fresh and dry nuts with or without the seed coat
}

\author{
Iskender Arcan, Ahmet Yemenicioğlu* \\ Department of Food Engineering, Faculty of Engineering, Izmir Institute of Technology, 35430, Gülbahçe Köyü, Urla, İmir, Turkey
}

\section{A R T I C L E I N F O}

\section{Article history:}

Received 28 December 2007

Received in revised form 9 September 2008

Accepted 20 October 2008

\section{Keywords:}

Hazelnut

Walnut

Pistachio

Tea

Antioxidant activity

Phenolic content

Food analysis

Food composition

\begin{abstract}
A B S T R A C T
Total antioxidant activities based on ABTS free radical scavenging activity and phenolic content of fresh or dry hazelnuts, walnuts and pistachios assayed with their seed coats changed between 3063 and $11,076 \mu \mathrm{mol}$ trolox equivalents/100 g d.w. and 256 and $755 \mathrm{mg}$ gallic acid equivalents/100 g d.w., respectively. The walnuts used in this study showed the highest antioxidant activity, followed by pistachios and hazelnuts. The removal of seed coat reduced the total antioxidant activity of hazelnuts, walnuts and pistachios almost 36, 90 and 55\%, respectively. The total antioxidant activities of investigated fresh and dry nuts are not considerably different. However, phenolic content and antioxidant activity in hydrophilic and ethanolic fractions obtained by successive extraction of nuts showed some variation. The antioxidant activity in 1-serving portion of fresh or dry walnuts is equivalent to that in almost 2-serving portions of black tea, and 1.2-1.7-serving portions of green and Earl Grey tea. One-serving portions of dry hazelnuts and fresh or dry pistachios contained antioxidant activity equivalent to that in $0.7-1$-serving portions of black tea. The antioxidant activity measurements correlated with phenolic content $\left(r^{2}=0.70\right)$. This study showed the potential of using fresh or dry nuts to develop functional foods with high antioxidant activity.
\end{abstract}

(c) 2009 Elsevier Inc. All rights reserved.

\section{Introduction}

Since antioxidants have shown protective effects in different diseases including cardiovascular disease, cancer, aging, and cataracts, extensive studies have been conducted concerning their relationship to food (Dashpande et al., 1996). Among the antioxidants, phenolic compounds have attracted a particular interest since foods containing varying amounts and forms of these compounds show different antioxidant potentials. Recently, studies related to the antioxidant potential of nuts such as hazelnuts, walnuts and pistachios showed that these foods are good sources of antioxidant phenolic compounds (Fukuda et al., 2003; Kornsteiner et al., 2006; Tokuşoğlu et al., 2005; Yurttas et al., 2000; Anderson et al., 2001; Gunduc and El, 2003). The clinical studies suggested that the supplementation of human diet with hazelnuts, walnuts or pistachios is beneficial for the amelioration of blood plasma antioxidant potential and lipid profiles that are closely related to cardiovascular risks (Edwards et al., 1999; Durak et al., 1999; Tapsell et al., 2004; Koçyigit et al., 2006; Lavedrine et al., 1999). The consumption of the oils of the indicated nuts also has beneficial effects on improvement of blood lipid profiles and/or

\footnotetext{
* Corresponding author. Tel.: +90 232 7506292; fax: +90 2327506196 .

E-mail address: ahmetyemenicioglu@iyte.edu.tr (A. Yemenicioğlu).
}

reduction of lipid peroxide levels (Lavedrine et al., 1999; Balkan et al., 2003; Zibaeenezhad et al., 2003; Hatipoğlu et al., 2004). Recently, some of the health-related phenolic compounds in nuts have been identified. For example, it was reported that walnuts are good sources of ellagic acid and ellagitannins, cancer chemopreventive polyphenolic compounds found only in limited numbers of fruits and nut species (Fukuda et al., 2003; Daniel et al., 1989; Clifford and Scalbert, 2000; Colaric et al., 2005). Tokuşoğlu et al. (2005) reported that pistachios contain resveratrol, a cardioprotective and chemopreventive polyphenolic compound (Pervaiz, 2004; Kris-Etherton et al., 2002).

In the literature there are various studies related to the phenolic content and/or antioxidant activity of dry nuts (Fukuda et al., 2003; Kornsteiner et al., 2006; Yurttas et al., 2000; Gunduc and El, 2003; Colaric et al., 2005; Moure et al., 2001). However, it is difficult to compare the results obtained in these studies since they employed different extraction methods, reported phenolic content and antioxidant activities as equivalents of different compounds and/or used different antioxidant activity determination methods (Yurttas et al., 2000; Gunduc and El, 2003; Moure et al., 2001). Studies related to contribution of seed coat on antioxidant activity of nuts and antioxidant potential of fresh nuts are also scarce. Following harvest, most of the nuts are dried and stored until they are used in consumption or processing. A considerable number of nuts are also harvested at less ripe stages 
and consumed or processed fresh during the season. Drying prevents the microbial spoilage of nuts, but it can also cause some enzymatic or nonenzymatic oxidative changes that modify the phenolic compounds.

In this study, the phenolic content and free radical scavengingbased antioxidant activities of selected fresh and dry nuts were compared with each other and with those of different types of tea, which are the most popular sources of antioxidants, using the same extraction and assay conditions. The importance of the seed coat on the antioxidant activity of nuts has also been investigated by using fresh samples. The results of this work help better understand the antioxidant activity of fresh and dry nuts with or without their seed coat.

\section{Materials and methods}

\subsection{Materials}

The fresh and dry hazelnuts and walnuts (both fresh samples were with shells), dry pistachios and commercial tea samples (all in $2 \mathrm{~g}$ bags) were obtained from market places and supermarkets in Izmir (Turkey). The fresh pistachios with shells were obtained from a marketplace in Nicosia (Cyprus). The certified organically grown dry hazelnuts and walnuts were from Işık Organik Gıda A.Ș in Izmir (Turkey). The dry nuts (all unroasted) were used without removing their seed coat, while fresh samples were used with or without removing seed coats. The seed coats of samples were removed manually by hand just before extraction. ABTS (2,2'-azino-bis-(3ethylbenz-thiazoline-6-sulfonic acid)) was purchased from Sigma Chem. Co. (St. Louis, Mo., USA). Trolox (6-hydroxy-2,5,7,8-tetramethylchroman-2-carboxylic acid) was purchased from Fluka (Switzerland).

\subsection{Determination of moisture content of nuts}

The moisture content of samples was determined by oven drying method (oven temperature $=103 \pm 2{ }^{\circ} \mathrm{C}$ ) of ISO 665-2000 for oilseeds given in UNECE standard No. DF-04 (2000).

\subsection{Extraction of antioxidant compounds in nuts}

The extraction of antioxidant compounds was conducted by homogenization of a $20 \mathrm{~g}$ sample (fresh nuts with or without seed coat, dry nuts with seed coat) with $100 \mathrm{~mL}$ of cold water $\left(4^{\circ} \mathrm{C}\right)$ in a Waring blender for $2 \mathrm{~min}$. The homogenate was transferred to a glass cylinder and the residues left in the blender jar were collected and added to the main homogenate by washing with $20 \mathrm{~mL}$ of additional cold water. The slurry obtained was then further homogenized in a disperser-homogenizer (IKA, DI 18, Basic, Brasil) at $18,000 \mathrm{rpm}$ for $2 \mathrm{~min}$ and centrifuged at $15,000 \times \mathrm{g}$ at $4{ }^{\circ} \mathrm{C}$ for $15 \mathrm{~min}$. The fatty layer at the top of the supernatant was discarded and the supernatant and pellet were collected. The collected supernatant ("aqueous extract") was filtered and kept in an icewater bath until it was assayed for antioxidant activity and phenolic content. Meanwhile the pellet was suspended in $120 \mathrm{~mL}$ ethanol (96\%) and homogenized in the disperser-homogenizer at $18,000 \mathrm{rpm}$ for $4 \mathrm{~min}$. The extract was then clarified by centrifugation at $15,000 \times g$ at $4{ }^{\circ} \mathrm{C}$ for $15 \mathrm{~min}$. This extract ("ethanolic extract") was kept in an ice-water bath until it was assayed for antioxidant activity and phenolic content.

\subsection{Antioxidant activity of nuts}

The antioxidant activity of aqueous or ethanolic extracts of nuts was determined spectrophotometrically (Shimadzu, Model 2450, Japan) according to the method of Re et al. (1999) as trolox equivalents by monitoring ABTS free radical cation decolorization caused by test samples at $734 \mathrm{~nm}$. The reaction mixture was formed by mixing $2 \mathrm{~mL}$ potassium persulfate oxidized ABTS free radical solution in phosphate buffered saline (PBS) at $\mathrm{pH} 7.4$ and 5,10 and $15 \mu \mathrm{L}$ of extract (or $20 \mu \mathrm{L}$ of trolox (0.01-0.03 $\mu \mathrm{mol}$ in reaction mixture) used to prepare the standard curve). The decrease in absorbance was monitored for $10 \mathrm{~min}$. The results were calculated as area under the curve (AUC) values and expressed as $\mu \mathrm{mol}$ trolox equivalents per $100 \mathrm{~g}$ fresh or dry weight of samples, or $\mu \mathrm{mol}$ trolox equivalents per serving portion of samples. One suggested serving portion of nuts is $42 \mathrm{~g}$ (or 1.5 ounce) according to the "Dietary Approaches to Stop Hypertension (DASH)" eating plan reported by USDHHS (2005). In this study the serving portion was based on fresh weight of samples containing seed coat. To calculate the AUC, the percent inhibition/concentration values for the extracts and trolox were plotted separately against test periods (1, 3, 6, $10 \mathrm{~min}$ ). The division of the areas of curves for each extract to that of trolox was used to calculate the AUC value. All sample extracts were tested three times at three different volumes.

\subsection{Phenolic content of nuts}

The phenolic content was determined according to the method of Singleton and Rossi (1965) by using Folin-Ciocalteu as reactive reagent. A $1 \mathrm{~mL}$ of appropriately diluted sample was mixed with $5 \mathrm{~mL}$ of $1 / 10$ diluted Folin-Ciocalteu solution. After $3 \mathrm{~min}$ incubation, $4 \mathrm{~mL} 7.5 \% \mathrm{Na}_{2} \mathrm{CO}_{3}$ was added to the mixture and the absorbance of the sample was determined by a spectrophotometer at $765 \mathrm{~nm}$ after it was incubated for $2 \mathrm{~h}$ at room temperature. Deionized water and ethanol (diluted as sample) were used as control. The average of triplicate measurements was used to calculate the phenolic content as mg gallic acid equivalents per $100 \mathrm{~g}$ fresh or dry weight of samples or mg gallic acid equivalents per serving portion (defined in Section 2.4) of samples. Gallic acid was used for the preparation of standard curve.

\subsection{Antioxidant activity and phenolic content of tea samples}

A 1-serving portion of commercial green, black and Earl Grey tea was prepared by slightly modifying the method described by Lee et al. (2003). Commercial tea bags containing $2 \mathrm{~g}$ tea were immersed into $200 \mathrm{~mL}$ hot water at $95^{\circ} \mathrm{C}$ for $3 \mathrm{~min}$. The samples were cooled, centrifuged at $12,000 \mathrm{~g}$ at $4{ }^{\circ} \mathrm{C}$ for $5 \mathrm{~min}$ and assayed for their antioxidant activity and phenolic content as described in Sections 2.4 and 2.5, respectively. The results were expressed as $\mu \mathrm{mol}$ trolox or mg gallic acid equivalents per serving portion.

\subsection{Statistical analysis}

The statistical analysis was conducted by analyzing data for the analysis of variance. Values are significantly different at $P<0.05$ as determined by Fisher's protected least significant difference.

\section{Results and discussion}

\subsection{Antioxidant activity of fresh nuts with or without seed coat}

To avoid possible changes in phenolic compounds of dry nuts, the contribution of seed coat in phenolic content and antioxidant activity of nuts was determined by using fresh samples (Figs. 1 and 2 ). As seen in Table 1, the moisture content of fresh nuts was $\geq 29 \%$, whereas all other dry nuts contain $\leq 5 \%$ moisture. In fresh hazelnuts, the presence of seed coat did not affect the phenolic content of aqueous extracts significantly $(P>0.05)$, but a slight increase in antioxidant activity occurred when the seed coat was 


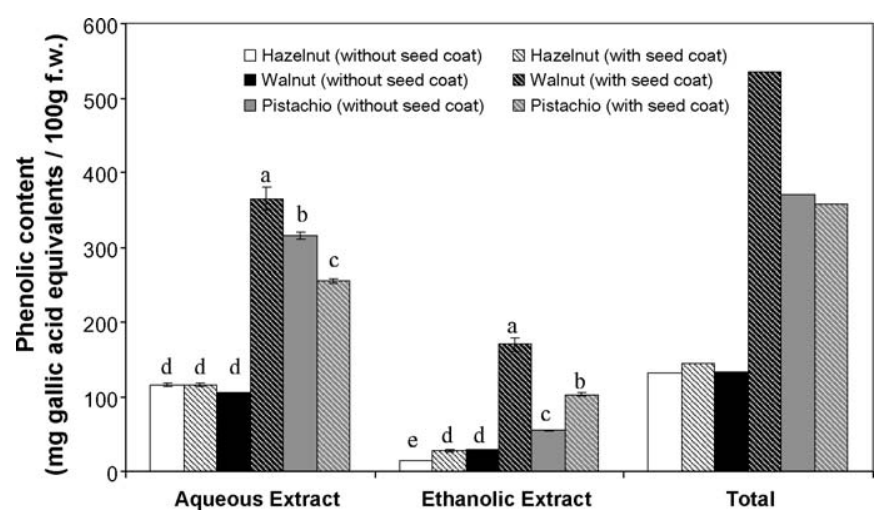

Fig. 1. Phenolic content in different extracts of fresh nuts with or without the seed coat (the data for aqueous and ethanolic extracts are reported as mean \pm standard deviation; total values are sum of average measurements for aqueous and ethanolic extracts).

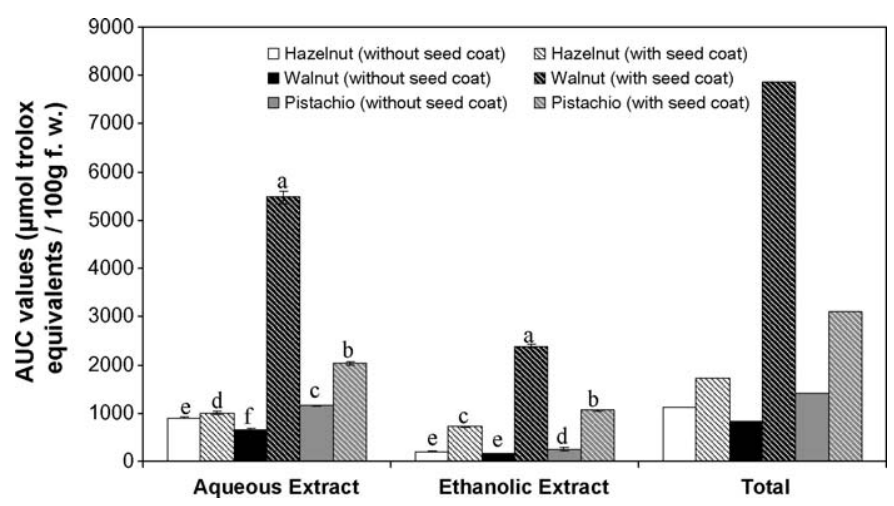

Fig. 2. Antioxidant activities in different extracts of fresh nuts with or without the seed coat (the data for aqueous and ethanolic extracts are reported as mean \pm standard deviation; total values are sum of average measurements for aqueous and ethanolic extracts).

Table 1

Some characteristics of hazelnuts, walnuts and pistachios used in this study (all samples with intact seed coat).

\begin{tabular}{llc}
\hline Product & Characteristics & Moisture content $^{\mathrm{a}}(\mathrm{g} / 100 \mathrm{~g})$ \\
\hline Hazelnut & Fresh & 44.0 \\
Hazelnut & Dry & 4.2 \\
Hazelnut & Dry, organic & 5.1 \\
Walnut & Fresh & 29.0 \\
Walnut & Dry & 4.9 \\
Walnut & Dry, organic & 5.1 \\
Pistachio & Fresh & 35.0 \\
Pistachio & Dry & 3.1 \\
\hline
\end{tabular}

a The moisture content was the average of two determinations on test samples. not removed from the nuts. In contrast, the phenolic content and antioxidant activity of aqueous extracts from fresh walnuts increased almost 3.5- and 8.4-fold, respectively, when these nut samples were extracted without the seed coat having been removed. This result showed the significant amounts of antioxidant phenolic compounds in the seed coat of fresh walnuts. Fukuda et al. (2003) reported the major antioxidant phenolic compounds in walnuts as ellagitanins. According to Colaric et al. (2005), the concentrations of abundant phenolic compounds, juglone, syringic and ellagic acid, in walnut seed coat (pellicle) are over 20-fold higher than the concentrations of these compounds in the seed. In fresh pistachios with the seed coat, the antioxidant activity of aqueous extract was 1.8-fold higher than that of fresh pistachios without the seed coat. However, the phenolic content in aqueous extracts reduced significantly $(P<0.05)$ when pistachios were extracted without the seed coat having been removed. This result suggested the low content but high reactivity of aqueous seed coat phenolics in pistachios.

In all nuts, the ethanolic extracts contained lower amounts of phenolic compounds than the aqueous extracts obtained before ethanol extraction. In fresh hazelnuts and pistachios, the phenolic content and antioxidant activity of ethanolic extracts reduced almost 2- and 4-fold when seed coat was removed from the nuts, respectively. The fresh walnuts with seed coat contained the highest amount of ethanol soluble phenolic compounds. It is clear that the antioxidant phenolics are concentrated in the seed coat of walnuts, since the removal of seed coat of these nuts caused an almost 15-fold reduction in antioxidant activity of ethanolic extracts. The total antioxidant activities (sum of average antioxidant activities in aqueous and ethanolic extracts) better reflected the importance of seed coat in antioxidant activity of different nuts. In fresh hazelnuts, walnuts and pistachios the removal of seed coat reduced the total antioxidant activity obtained from these nuts almost 36, 90 and 55\%, respectively. The removal of the seed coat reduced the total phenolic content of fresh walnuts by almost $75 \%$, while total amounts of phenolic compounds in fresh hazelnuts and pistachios increased or reduced only by 9 and 3\% after removal of seed coat, respectively.

\subsection{Antioxidant activity of dry nuts}

The phenolic content and antioxidant activity of dry nuts used in this study are given in Table 2 . On dry weight basis, the phenolic content and antioxidant activity in aqueous extracts of dry walnuts and pistachios were significantly (1.2-1.5-fold) lower than those of fresh walnuts and pistachios $(P<0.05)$. The ethanolic extracts of dry walnuts also had lower phenolic content and antioxidant activity than those of fresh walnuts. In contrast, ethanolic extracts of dry pistachios contained 1.3-1.4-fold higher phenolic content

Table 2

Phenolic content and antioxidant activities of fresh and dry hazelnuts, walnuts and pistachios containing the seed coat.

\begin{tabular}{|c|c|c|c|c|c|c|}
\hline \multirow[t]{2}{*}{ Product } & \multicolumn{3}{|c|}{ Phenolic content (mg gallic acid equivalents/100 g d.w.) } & \multicolumn{3}{|c|}{ AUC value ( $\mu$ mol trolox equivalents/100 g d.w.) } \\
\hline & Aqueous extract ${ }^{a}$ & Ethanolic extract & Total $^{\mathrm{b}}$ & Aqueous extract & Ethanolic extract & Total \\
\hline Fresh hazelnut & $207 \pm 3 \mathrm{e}$ & $49 \pm 2 d$ & $256(61)^{c}$ & $1783 \pm 51 \mathrm{e}$ & $1280 \pm 8 \mathrm{f}^{\mathrm{d}}$ & $3063(723)$ \\
\hline Dry hazelnut & $386 \pm 40 b$ & $39 \pm 0.5 \mathrm{e}$ & $425(171)$ & $3078 \pm 68 c$ & $495 \pm 16 \mathrm{~h}$ & $3573(1437)$ \\
\hline Dry hazelnut (organic) & $315 \pm 4 c$ & $56 \pm 1 \mathrm{~d}$ & $371(148)$ & $2592 \pm 22 d$ & $882 \pm 17 \mathrm{~g}$ & $3474(1385)$ \\
\hline Fresh walnut & $515 \pm 21 \mathrm{a}$ & $240 \pm 13 a$ & $755(225)$ & $7713 \pm 176 a$ & $3363 \pm 41 a$ & $11,076(3300)$ \\
\hline Dry walnut & $414 \pm 18 b$ & $175 \pm 17 c$ & $589(235)$ & $6498 \pm 77 b$ & $2555 \pm 196 c$ & $9053(3612)$ \\
\hline Dry walnut (organic) & $348 \pm 22 c$ & $190 \pm 4 b$ & $538(215)$ & $6327 \pm 238 b$ & $3190 \pm 94 b$ & 9517 (3792) \\
\hline Fresh pistachio & $390 \pm 5 b$ & $157 \pm 3 c$ & $547(150)$ & $3111 \pm 57 c$ & $1627 \pm 19 \mathrm{e}$ & $4738(1301)$ \\
\hline Dry pistachio & $245 \pm 4 d$ & $216 \pm 16 b$ & $461(188)$ & $2319 \pm 41 \mathrm{~d}$ & $2124 \pm 78 d$ & 4443 (1808) \\
\hline
\end{tabular}

a The data for aqueous and ethanolic extracts were reported as mean \pm standard deviation.

b Total values are sum of average measurements for aqueous and ethanolic extracts.

c The numbers in the parenthesis indicate the total phenolic content or AUC values per serving portions of nuts (42 $\mathrm{g}$ based on fresh weight).

d Values with different letters in the columns are significantly different $(P<0.05)$. 
and antioxidant activity than those of fresh pistachios. The phenolic content and antioxidant activity of aqueous extracts of dry hazelnuts were also at least 1.5-fold higher than those of the fresh hazelnuts. The phenolic content in ethanolic extracts of dry hazelnuts and fresh hazelnuts were not considerably different. In fact, there is also no statistically significant difference between ethanol soluble phenolic content of fresh hazelnuts and dry organic hazelnuts $(P>0.05)$. However, antioxidant activity of ethanolic extracts of dry hazelnuts was 1.5-2.5-fold lower than that of fresh hazelnuts. The differences also exist between the total phenolic content of dry and fresh hazelnuts and walnuts. In contrast, although there are possible differences in cultivar, growth conditions and location as well as post-harvest handling of nuts used in this study, the differences between total antioxidant activities of dry and fresh nuts did not exceed 1.2-fold and varied within a narrow range. The differences between total antioxidant activities of conventional and organic nuts (walnuts and hazelnuts) used in this work were also negligible $(<1.1$-fold). In this study, the total antioxidant activity refers to the sum of antioxidant activity solubilized by water and ethanol. However, there are also insoluble phenolic antioxidants bound to plant cell wall carbohydrates (Beveridge et al., 2000). In fact, Serpen et al. (2007) recently developed a method for determination of insoluble antioxidant activity and emphasized the important contribution of insoluble antioxidants bound by dietary fiber components. Thus, further studies are needed to evaluate the insoluble antioxidant activity in different nuts which are rich sources of dietary fiber. The total phenolic content of dry pistachios and hazelnuts determined in this study is very close to the previously reported ranges of phenolic content in dry pistachios (492-1442 mg gallic acid equivalents/100 g f.w.) and dry hazelnuts (101-433 mg gallic acid equivalents/100 g f.w.) (Kornsteiner et al., 2006). The dry walnuts used in this study, on the other hand, had lower phenolic content than the dry walnuts (1020-2052 mg gallic acid equivalents/100 g f.w.) investigated by Kornsteiner et al. (2006) (values reported for dry nuts as dry weight in Table 2 are 3-5\% lower if expressed as fresh weight).

\subsection{Antioxidant activity of nuts as compared with those of tea samples}

To better evaluate their contribution to human diet, total antioxidant activity (total AUC values) and total phenolic content of 1-serving portions ( $42 \mathrm{~g}$ ) of nuts were compared with those of 1serving portions $(200 \mathrm{~mL})$ of green, black and Earl Grey tea, the most popular sources of antioxidants. From different tea samples, green tea showed the highest antioxidant activity, while Earl Grey tea and black tea ranked second and third in antioxidant activity, respectively (Table 3 ). Green tea and Earl Grey tea had quite similar phenolic contents $(P>0.05)$, while black tea showed 1.6-fold lower phenolic content than those samples. The comparison of results for nut and tea samples indicated that the total antioxidant

Table 3

Phenolic content and antioxidant activities of 1-serving portion of different tea samples.

\begin{tabular}{|c|c|c|}
\hline Product & $\begin{array}{l}\text { Phenolic content }^{\mathrm{a}} \\
\text { (mg gallic acid equivalents/ } \\
\text { 1-serving portion }{ }^{\mathrm{b}} \text { ) }\end{array}$ & $\begin{array}{l}\text { AUC value ( } \mu \mathrm{mol} \\
\text { trolox equivalents/ } \\
\text { 1-serving portion) }\end{array}$ \\
\hline Black tea & $87 \pm 2 a^{c}$ & $1754 \pm 97 a$ \\
\hline Earl Grey tea & $137 \pm 2 b$ & $2264 \pm 55 b$ \\
\hline Green tea & $140 \pm 5 b$ & $2789 \pm 99 c$ \\
\hline
\end{tabular}

\footnotetext{
a The data were reported as mean \pm standard deviation.

b $200 \mathrm{~mL}$ (see methods section for preparation of tea samples).

c Values with different letters in the columns are significantly different $(P<0.05)$
}

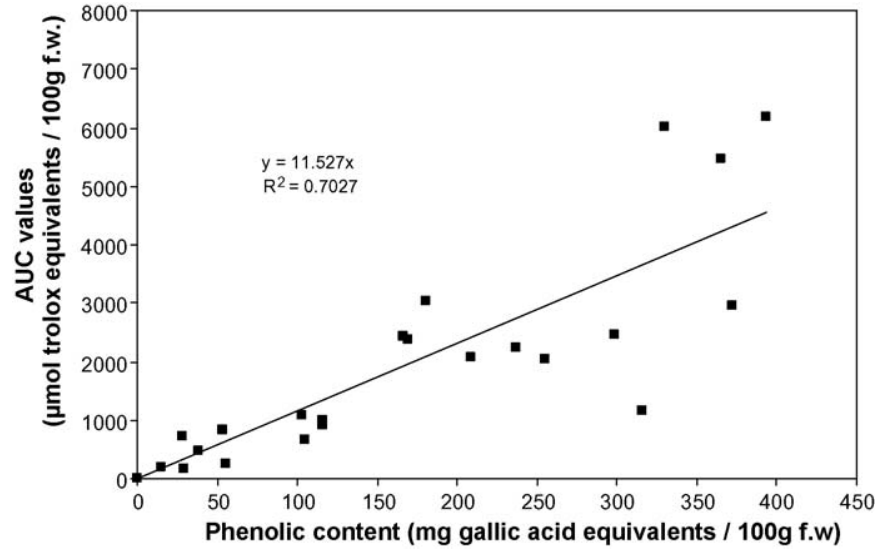

Fig. 3. Relationship between antioxidant activity and phenolic content of nuts $(n=22)$.

activity contained by a 1-serving portion of fresh or dry walnuts is equivalent to that in 1.5-1.7, and 1.2-1.3-serving portions of Earl Grey and green tea, respectively. A 1-serving portion of walnuts also contained antioxidant activity equivalent to that in almost 2serving portions of black tea. The total amounts of phenolic compounds in 1-serving portion of walnuts were also 1.5-2.7-fold higher than those in 1-serving portion of different tea samples. A 1serving portion of dry hazelnuts, and fresh or dry pistachios, on the other hand, contained phenolic content and antioxidant activities equivalent to those in 1.7-2.0 and 0.7-1.0-serving portions of black tea, respectively. However, 1-serving portion of fresh hazelnuts contained 1.4- and 2.4-fold lower phenolic content and antioxidant activity than did 1-serving portion of black tea.

\subsection{Relationship between antioxidant activity and phenolic content of nuts}

The plotting of results of antioxidant activity (AUC values) vs. phenolic content of aqueous and ethanolic extracts showed the positive correlation between these parameters $\left(r^{2}=0.70\right)$ (Fig. 3 ). The correlation found in this study between antioxidant activity and phenolic content was lower than that between the same parameters in different tomato lines $\left(r^{2}>0.90\right)$ (Hanson et al., 2004), phenolic-rich food including cocoa, black tea, green tea and red wine $\left(r^{2}=0.98\right)$ (Lee et al., 2003) and olive oil samples $\left(r^{2}=0.86\right)$ (Sánchez et al., 2007). Teow et al. (2007) reported a quite similar correlation between antioxidant activity and phenolic content $\left(r^{2}=0.69\right)$ of different types of sweet potatoes, whereas Tawaha et al. (2007) reported slightly higher correlations for different extracts of selected plant species $\left(r^{2}=0.72\right.$ and 0.79$)$. All these studies were conducted by testing antioxidant activity and phenolic content with the ABTS and Folin-Ciocalteu methods, respectively. However, they used different materials and various extraction methods to obtain the phenolic compounds.

\section{Conclusions}

The results of this study clearly showed the importance of seed coat for antioxidant activity of nuts. The removal of seed coat considerably reduced the total antioxidant activity obtained from hazelnuts, walnuts and pistachios used in this study. Although there are possible differences in cultivars, growth conditions and location, and postharvest handling of nuts used in this study, the total antioxidant activities of fresh and dry nuts (organic or not) are not considerably different. However, amounts of phenolic compounds and their resulting antioxidant activity in hydrophilic and hydrophobic fractions show some variations. The antioxidant 
activities of nuts correlate well with phenolic content. The phenolic content and antioxidant activity in 1-serving portions of nuts are comparable to those in 1-serving portions of green, black or Earl Grey tea used in this study. This work clearly showed the good potential of using fresh or dry nuts to develop novel nut products having beneficial effects on human health.

\section{References}

Anderson, K., Teuber, S.S., Gobeille, A., Cremin, P., Waterhouse, A.L., Steinberg, M., 2001. Walnut polyphenolics inhibit in vitro human plasma and LDL oxidation. Journal of Nutrition 131, 2837-2842.

Balkan, J., Hatipoğlu, A., Aykaç-Toker, G., Uysal, M., 2003. Influence on hazelnut oil administration on peroxidation status of erythrocytes and apolipoprotein B 100-containing lipoproteins in rabbits fed on a high cholesterol diet. Journal of Agricultural and Food Chemistry 51, 3905-3909.

Beveridge, T., Loubert, E., Harrison, J.E., 2000. Simple measurement of phenolic esters in plant cell walls. Food Research International 33, 775-783.

Clifford, M.N., Scalbert, A., 2000. Ellagitannins - nature, occurrence and dietary burden. Journal of the Science of Food and Agriculture 80, 1118-1125.

Colaric, M., Veberic, R., Solar, A., Hudina, M., Stampar, F., 2005. Phenolic acids, syringaldehyde, and juglone in fruits of different cultivars of Juglans regia $\mathrm{L}$. Journal of Agricultural and Food Chemistry 53, 6390-6396.

Daniel, E.M., Krupnick, A.S., Heur, Y., Blinzler, J.A., Nims, R.W., Stoner, G.D., 1989. Extraction stability, and quantitation of ellagic acid in various fruits and nuts. Journal of Food Composition and Analysis 2, 338-349.

Dashpande, S.S., Dashpande, U.S., Salunkhe, D.K., 1996. Nutritional and health aspects of food antioxidants. In: Madhavi, D.L., Dashpande, S.S., Salunkhe, D.K. (Eds.), Food Antioxidants. Marcel Dekker Inc, New York, pp. 361-469.

Durak, I., Köksal, I., Kaçmaz, M., Büyükkoçak, S., Çimen, B.M.Y., Öztürk, H.S., 1999. Hazelnut supplementation enhances plasma antioxidant potential and lowers plasma cholesterol levels. Clinica Chimica Acta 284, 113-115.

Edwards, K., Kwaw, I., Matud, J., Kurtz, I., 1999. Effect of pistachio nuts on serum lipid levels in patients with moderate hypercholesterolemia. Journal of the American College of Nutrition 18, 229-232.

Fukuda, T., Ito, H., Yoshida, T., 2003. Antioxidative polyphenols from walnuts (Juglas regia L.). Phytochemistry $63,795-801$.

Gunduc, N., El, S.N., 2003. Assessing antioxidant activities of phenolic compounds of common Turkish food and drinks on in vitro low-density lipoprotein oxidation. Journal of Food Science 68, 2591-2595.

Hanson, P.M., Yang, R., Wu, J., Chen, J., Ledesma, D., Tsou, S.C.S., 2004. Variation for antioxidant activity and antioxidants in tomato. Journal of the American Society for Horticultural Science 129, 704-711.

Hatipoğlu, A., Kanbağlı, Ö., Balkan, J., Küçük, M., Çavikbaş, U., Aykaç-Toker, G., Berkkan, H., Uysal, M., 2004. Hazelnut oil administration reduces aortic cholesterol accumulation and lipid peroxides in the plasma, liver, and aorta of rabbits fed a high-cholesterol diet. Bioscience Biotechnology and Biochemistry 68, 2050-2057.

Kris-Etherton, P.M., Hecker, K.D., Bonanome, A., Coval, S.M., Binkoski, A.E., Hilpert, K.F., Griel, A.E., Etherton, T.D., 2002. Bioactive compounds in foods: Their role in the prevention of cardiovascular disease and cancer. The American Journal of Medicine 113, 71-88.
Kocyigit, A., Koylu, A.A., Keles, H., 2006. Effect of pistachio nuts consumption on plasma lipid profile and oxidative status in healthy volunteers. Nutrition, Metabolism and Cardiovascular Diseases 16, 202-209.

Kornsteiner, M., Wagner, K., Elmadfa, I., 2006. Tocopherols and total phenolics in 10 different nut types. Food Chemistry 98, 381-387.

Lavedrine, F., Zmirou, D., Ravel, A., Balducci, F., Alary, J., 1999. Blood cholesterol and walnut consumption: a cross-sectional survey in France. Preventive Medicine $28,333-339$.

Lee, K.W., Kim, Y.J., Lee, H.J., Lee, C.Y., 2003. Cocoa has more phenolic phytochemicals and a higher antioxidant capacity than teas and red wine. Journal of Agricultural and Food Chemistry 51, 7292-7295.

Moure, A., Franco, D., Sineiro, J., Dominguez, H., Jose Nunez, M., Lema, J.M., 2001. Antioxidant activity of extracts from Gevuina avellana and Rosa rubiginosa defatted seeds. Food Research International 34, 103-109.

Pervaiz, S., 2004. Chemotherapeutic potential of the chemopreventive phytoalexin resveratrol. Drug Resistance Updates 7, 333-344.

Re, R., Pellegrini, N., Proteggente, A., Pannala, A., Yang, M., Rice-Evans, C., 1999. Antioxidant activity applying an improved ABTS radical cation decolorization assay. Free Radical Biology and Medicine 26, 1231-1237.

Serpen, A., Capuano, E., Fogliano, V., Gökmen, V., 2007. A new procedure to measure the antioxidant activity of insoluble food components. Journal of Agricultural and Food Chemistry 55, 7676-7681.

Sánchez, C.S., Gonzalez, A.M.T., Garcia-Perrilla, M.C., Granados, J.J.Q., de la Serrana H.L.G., Martiez, M.C.L., 2007. Different radical scavenging tests in virgin olive oil and their relation to the total phenol content. Anlytica Chimica Acta 593, $103-$ 107.

Singleton, V.L., Rossi, J.A., 1965. Colorimetry of total phenolics with phosphomolibdic-phosphotungstic acid reagents. American Journal of Enology and Viticulture 16, 144-158.

Tapsell, L.C., Gillen, L.J., Patch, C.S., Batterham, M., Owen, A., Bare, M., Kennedy, M. 2004. Including walnuts in a low-fat/modified-fat diet improves HDL cholesterol-to-total cholesterol ratios in patients with type 2 diabetes. Diabetes Care 27, 2777-2783.

Tawaha, K., Alali, F.Q., Gharaibeh, M., Mohammad, M., El-Elimat, T., 2007. Antioxidant activity and total phenolic content of selected Jordanian plant species. Food Chemistry 104, 1372-1378.

Teow, C.C., Truong, V., McFeeters, R.F., Thompson, R.L., Pecota, K.V., Yencho, G.C. 2007. Antioxidant activities, phenolic and b-carotene contents of sweet potato genotypes with varying flesh colors. Food Chemistry 103, 829-838.

Tokuşoğlu, Ö., Ünal, M.K., Yemis, F., 2005. Determination of the phytoalexin resveratrol (3,5,4'-trihydroxystilbene) in peanuts and pistachios by high-performance liquid chromatographic diode array (HPLC-DAD) and gas chromatography-mass spectrometry (GC-MS). Journal of Agricultural and Food Chemistry 53, 5003-5009.

UNECE (United Nations Economic Commission for Europe) Standard for Hazelnut Kernels, 2000. Determination of the moisture content for dry produce (nuts) Standard No: DF-04, pp. 7-8.

USDHHS (United States Department of Health and Human Services), USDA (United States Department of Agriculture), 2005. Dietary Guidelines for Americans, 6th ed. Government Printing Office, Washington, D.C..

Yurttas, H.C., Schafer, H.W., Warthesen, J.J., 2000. Antioxidant activity of nontocopherol hazelnut (Corylus spp.) phenolics. Journal of Food Science 65, 276-280.

Zibaeenezhad, M.J., Rezaiezadeh, M., Mowla, A., Ayatollahi, S.M., Panjehshahin, M.R. 2003. Antihypertriglyceridemic effect of walnut oil. Angiology 54, 411-414. 